\title{
Utilização do planejamento experimental no estudo do efeito da composição de misturas de bentonitas na reologia de fluidos de perfuração. Parte I: composições binárias
}

\section{(Experimental design applied to the study of composition effect of bentonite on the rheology of drilling fluids.Part I: binary compositions)}

\author{
L. F. A. Campos, L. V. Amorim, H. C. Ferreira \\ Departamento de Eng. de Materiais, Universidade Federal de Campina Grande \\ Av. Aprígio Veloso 882, Campina Grande, PB 58109-970 \\ liszandra@labdes.ufcg.edu.br
}

\begin{abstract}
Resumo
O objetivo deste trabalho foi utilizar o planejamento experimental, aplicado ao estudo de misturas, para avaliar o efeito da composição de misturas binárias de bentonitas na reologia de fluidos de perfuração de poços de petróleo. Por meio do planejamento foram determinadas as proporções dos componentes nas misturas binárias das argilas e então ajustados modelos de regressão relacionando viscosidade aparente (VA), viscosidade plástica (VP) e volume de filtrado (VF) com a proporção de cada argila. A aplicação desta ferramenta permitiu delimitar uma faixa de composições que favorece a melhoria das propriedades reológicas e de filtração das dispersões estudadas.

Palavras-chave: bentonita, mistura de argilas, planejamento experimental, reologia, fluidos de perfuração.
\end{abstract}

Abstract

The purpose of this work was to apply experimental design to the study of composition effect binary mixtures of bentonite on the rheology of drilling fluids. Through the experimental design were defined the components proportions in the binary clays mixtures and then adjusted regression models relating the parameters, apparent and plastic viscosities and filtrate volume, with the proportion of each clay. The application of this tool allowed to delimit a strip of compositions that favors the improvement of the rheological properties of the dispersions of bentonite clays. Keywords: bentonite, clays mixtures, experimental design, rheology, drilling fluids.

\section{INTRODUÇÃO}

Os planejamentos experimentais para o estudo de misturas têm encontrado larga aplicação na ciência, na engenharia e particularmente na indústria [1-5]. A partir de um delineamento de misturas, a resposta ou propriedade muda somente quando são feitas alterações nas proporções dos componentes que fazem parte dessa mistura. Portanto, a finalidade principal de se utilizar essa metodologia é verificar como as respostas ou propriedades de interesse são afetadas pela variação das proporções dos componentes da mistura. Nesse caso, as proporções dos componentes $\left(\mathrm{X}_{\mathrm{i}}\right)$ não são independentes, pois sua soma deve totalizar $100 \%$ [1].

Com os resultados obtidos no delineamento de misturas, pode-se utilizar polinômios simplificados para relacionar a propriedade de interesse às diversas proporções utilizadas. Isso possibilita a previsão quantitativa das propriedades de qualquer formulação no sistema estudado, realizando somente alguns experimentos [6].

Este procedimento pode ser particularmente útil quando se pretende desenvolver um determinado produto com propriedades específicas. Além das equações de regressão obtidas para cada propriedade especificada, são definidas metas e exigências para cada uma (geralmente inequações que exprimem a gama de variação admitida), de acordo com as especificações do tipo de produto a ser fabricado, sendo obtido um sistema de inequações ou equações simultâneas, geralmente não lineares. A resolução desse sistema conduz à definição de composições (misturas das mesmas matérias-primas) mais adequadas que atendam às propriedades especificadas para a aplicação. A resolução do sistema pode ser obtida por otimização matemática [7].

A mistura de matérias-primas é de fundamental importância para os diversos setores tecnológicos [1, 8-11]. A partir do conhecimento prévio das propriedades de cada componente original, bem como das misturas, em proporções definidas, é possível conhecer as características do produto 
industrializado, que por sua vez são fortemente dependentes das proporções usadas nas composições [12].

As bentonitas, argilas cujas propriedades são ditadas essencialmente pelos argilominerais esmectíticos, pertencem a um importante tipo de argilas largamente utilizado em muitos setores da indústria, como perfuração de poços de petróleo e de captação de água, fundições diversas, pelotização de minério de ferro, indústria química e farmacêutica, entre outros [13, 14].

Até o momento, na literatura sobre tecnologia de argilas, são encontrados poucos dados relativos ao estudo de misturas de argilas bentoníticas, contudo alguns pesquisadores [15, 16] vêm despertando o interesse nesta área.

Neste contexto, o presente trabalho tem como objetivo utilizar o planejamento experimental, aplicado ao estudo de misturas, para avaliar o efeito da composição de misturas binárias de bentonitas na reologia de fluidos de perfuração de poços de petróleo.

\section{MATERIAIS E MÉTODOS}

Foram utilizadas três amostras de argilas bentoníticas policatiônicas, extraídas da mina Bravo, localizada em Boa Vista, PB, identificadas como argila A, argila B e argila C.

Foi utilizado carbonato de sódio $\left(\mathrm{Na}_{2} \mathrm{CO}_{3}\right)$ anidro PA VETEC, para transformação das argilas individuais e das composições binárias de argilas bentoníticas em sódicas.

As amostras de argilas naturais foram secas em estufa a $60{ }^{\circ} \mathrm{C} \pm 2{ }^{\circ} \mathrm{C}$ durante um período de 7 dias. A seguir, foram moídas e passadas em peneira ABNT n. 200 (0,074 mm).

\section{Delineamento de misturas}

As composições binárias foram formuladas com as argilas A, $\mathrm{B}$ e $\mathrm{C}$ empregando a metodologia de modelagem de misturas do

Tabela I - Composições obtidas através do planejamento em rede simplex $\{2,2\}$, aumentado

[Table I - Compositions created by augmented $\{2,2\}$ simplex.]

\begin{tabular}{cccc}
\hline & \multicolumn{3}{c}{ Frações Mássicas } \\
\cline { 2 - 4 } Composições & Argila A & Argila B & Argila C \\
\hline 1 & 1 & 0 & 0 \\
2 & 0 & 1 & 0 \\
3 & 0 & 0 & 1 \\
4 & $3 / 4$ & $1 / 4$ & 0 \\
5 & $1 / 4$ & $3 / 4$ & 0 \\
6 & $1 / 2$ & $1 / 2$ & 0 \\
7 & 0 & $3 / 4$ & $1 / 4$ \\
8 & 0 & $1 / 4$ & $3 / 4$ \\
9 & 0 & $1 / 2$ & $1 / 2$ \\
10 & $3 / 4$ & 0 & $1 / 4$ \\
11 & $1 / 4$ & 0 & $3 / 4$ \\
12 & $1 / 2$ & 0 & $1 / 2$ \\
\hline
\end{tabular}

planejamento experimental [1]. Para definir as composições foi utilizado um planejamento em rede simplex $\{2,2\}$, aumentado. As combinações encontram-se na Tabela I.

As argilas individuais e as composições, nas proporções definidas (Tabela I), foram tratadas com solução concentrada de carbonato de sódio na proporção de $125 \mathrm{meq} / 100 \mathrm{~g}$ de argila seca e curadas por um período de 5 dias em câmara úmida. Após a cura, foram preparadas as dispersões de acordo com a metodologia descrita no item a seguir.

As dispersões em concentrações de 4,86\% em massa de argila, foram preparadas segundo a norma N-2605 [18], que consiste em adicionar 24,3 g de argila em $500 \mathrm{~mL}$ de água deionizada e agitar durante $20 \mathrm{~min}$ a uma velocidade de 17.000 rpm, em agitador mecânico Hamilton Beach, modelo 936. A seguir, as dispersões permaneceram em repouso por um período de $24 \mathrm{~h}$ em câmara úmida, com 100\% de umidade relativa.

O estudo reológico foi realizado segundo a norma N- 2605 [18], sendo determinadas as viscosidades aparente (VA) e plástica (VP) em viscosímetro Fann modelo 35A, e o volume de filtrado (VF) em filtro prensa da marca Fann.

Os dados reológicos foram obtidos em duplicatas; VA e VP são dadas em cP e VF é dado em mL.

$\mathrm{Na}$ representação do ajuste dos valores de resposta (VA, VP e VF) utilizou-se as equações linear (Equação A) e quadrática (Equação B).

$$
\begin{aligned}
& \mathrm{Y}\left(\mathrm{x}_{1}, \mathrm{x}_{2}\right)=\mathrm{b}_{1} \mathrm{x}_{1}+\mathrm{b}_{2} \mathrm{x}_{2} \\
& \mathrm{Y}\left(\mathrm{x}_{1}, \mathrm{x}_{2}\right)=\mathrm{b}_{1} \mathrm{x}_{1}+\mathrm{b}_{2} \mathrm{x}_{2}+\mathrm{b}_{12} \mathrm{x}_{1} \mathrm{x}_{2}
\end{aligned}
$$

sendo $\mathrm{Y}=$ estimativa da resposta $\mathrm{VA}, \mathrm{VP}$ e VF; $\mathrm{b}=$ coeficientes da equação determinados conforme CORNELL [13] e x = proporção dos componentes na mistura.

Os resultados obtidos no estudo reológico foram usados para calcular (iterativamente, até serem obtidos modelos estatisticamente significativos) os coeficientes das equações de regressão que relacionam VA, VP e VF com as proporções das argilas presentes nas dispersões.

Uma avaliação da eficiência dos modelos foi feita por meio de métodos estatísticos e comparação entre os resultados experimentais e os obtidos no modelo numérico. Todas as regressões foram obtidas com auxílio do software Statistica 6.0.

As equações de regressão foram sujeitas às restrições apresentadas pela norma da Petrobras [17] para fluidos à base de água e argila utilizados na perfuração de poços de petróleo, que são:

$$
\begin{aligned}
& V A / 15,0 \mathrm{cP} \\
& \mathrm{VP} / 4,0 \mathrm{cP} \\
& \mathrm{VF} \leq 18,0 \mathrm{~mL}
\end{aligned}
$$


A solução simultânea das equações de regressão, sujeitas a estas restrições, foi calculada usando o software Excel (Microsoft, 2002).

\section{RESULTADOS E DISCUSSÃO}

\section{Parâmetros reológicos}

As médias das duplicatas dos parâmetros VA, VP e VF das dispersões preparadas com as formulações (Tabela I) são apresentadas na Tabela II.

Tabela II - Valores médios de VA, VP e VF para as diferentes composições estabelecidas pelo planejamento em rede simplex $\{2,2\}$, aumentado.

[Table II - Average values of VA, VP and VF for different created compositions by augmented $\{2,2\}$ simplex.]

\begin{tabular}{ccccccc}
\hline Composições & Argila & Argila & Argila & VA & VP & VF \\
& A & B & C & $(\mathrm{cP})$ & $(\mathrm{cP})$ & $(\mathrm{mL})$ \\
\hline 1 & 1 & 0 & 0 & 8,3 & 3,5 & 20,0 \\
2 & 0 & 1 & 0 & 35,3 & 0,0 & 15,5 \\
3 & 0 & 0 & 1 & 19,8 & 1,5 & 21,0 \\
4 & $3 / 4$ & $1 / 4$ & 0 & 10,9 & 4,8 & 17,8 \\
5 & $1 / 4$ & $3 / 4$ & 0 & 19,3 & 5,3 & 14,7 \\
6 & $1 / 2$ & $1 / 2$ & 0 & 15,1 & 4,3 & 13,9 \\
7 & 0 & $3 / 4$ & $1 / 4$ & 71,0 & 0,0 & 18,9 \\
8 & 0 & $1 / 4$ & $3 / 4$ & 33,6 & 0,5 & 21,0 \\
9 & 0 & $1 / 2$ & $1 / 2$ & 25,5 & 0,2 & 17,3 \\
10 & $3 / 4$ & 0 & $1 / 4$ & 9,5 & 4,0 & 19,7 \\
11 & $1 / 4$ & 0 & $3 / 4$ & 8,2 & 3,5 & 17,5 \\
12 & $1 / 2$ & 0 & $1 / 2$ & 8,9 & 4,0 & 17,4 \\
\hline
\end{tabular}

A partir dos valores de VA, VP e VF apresentados na Tabela II foi possível gerar equações de regressão ou modelos, correlacionando as frações mássicas dos componentes originais com os parâmetros VA, VP e VF.

As Equações F, G e H correlacionando os valores de VA, VP e VF com as frações mássicas dos componentes A e B, desprezando os termos com coeficientes não significativos, são apresentadas a seguir:

$$
\begin{aligned}
& \mathrm{VA}(\mathrm{cP})=9,79 \mathrm{~A}+34,10 \mathrm{~B}-32,28 \mathrm{AB} \\
& \mathrm{VP}(\mathrm{cP})=2,97 \mathrm{~A}+13,74 \mathrm{AB} \\
& \mathrm{VF}(\mathrm{mL})=20,67 \mathrm{~A}+15,96 \mathrm{~B}-14,13 \mathrm{AB}
\end{aligned}
$$

sendo A a fração mássica da argila A e B a fração mássica da argila $\mathrm{B}$.

Através das Equações F, G e H observa-se que as frações mássicas das argilas A e B contribuem para o aumento de VA, VP e VF. Nas Equações F e H observa-se que os componentes A e B interagem sinergicamente (coeficiente das interações A e B é maior que zero), já na Equação $G$ estes componentes interagem antagonisticamente.

A Tabela III apresenta os parâmetros estatísticos para as Equações F, G e H ajustadas. Analisando os parâmetros estatísticos principais (Teste $\mathrm{F}$, Valor $\mathrm{p}$, coeficiente de múltipla determinação, $\mathrm{R}^{2}$ e coeficiente de múltipla determinação ajustado, $\mathrm{R}_{\mathrm{A}}{ }_{\mathrm{A}}$ ), observa-se que os modelos são estatisticamente significativos ao nível estipulado (valor $\mathrm{p} \leq$ nível de significância), que o $\mathrm{R}^{2}$ e o $\mathrm{R}_{\mathrm{A}}^{2}$ mostram que o modelo ajustado para VA não apresenta variabilidade considerável, e o modelo ajustado para VP apresenta pequena variabilidade; quanto mais perto da unidade estiver o valor de $\mathrm{R}^{2}$, melhor terá sido o ajuste do modelo aos dados observados.

Tabela III - Estatísticas relevantes para análise de variância das variáveis VA, VP e VF nas Equações F, G e H.

[Table III - Statistics properties relevant for variance analysis of the variables VA, VP and VF in Equations $F, G$ and $H$.]

\begin{tabular}{cccccc}
\hline Propriedade & Modelo & Teste $\mathrm{F}$ & Valor $\mathrm{p}$ & $\mathrm{R}^{2}$ & $\mathrm{R}_{\mathrm{A}}$ \\
\hline VA & Quadrático & 69,2532 & 0,0000 & 0,9584 & 0,9446 \\
$\mathrm{VP}$ & Quadrático & 10,6729 & 0,0105 & 0,7806 & 0,7074 \\
$\mathrm{VF}$ & Quadrático & 12,7040 & 0,0069 & 0,8090 & 0,7453 \\
\hline$R^{2}:$ coeficiente de múltipla determinação e & $R^{2}{ }_{A}: R^{2}$ ajustado. &
\end{tabular}

Em resumo, as Equações $\mathrm{F}, \mathrm{G}$ e $\mathrm{H}$ podem ser tomadas como sendo estatisticamente significativas para descrever o comportamento de VA, VP e VF respectivamente em função das frações mássicas das argilas A e B.

As Equações I, J e L correlacionando os valores de VA, VP e VF com as frações mássicas dos componentes A e C são apresentadas a seguir:

$$
\begin{aligned}
& \mathrm{VA}(\mathrm{cP})=10,37 \mathrm{~A}+17,14 \mathrm{C} \\
& \mathrm{VP}(\mathrm{cP})=3,36 \mathrm{~A}+1,69 \mathrm{C}+6,22 \mathrm{AC} \\
& \mathrm{VF}(\mathrm{mL})=21,00 \mathrm{~A}+20,20 \mathrm{C}-11,78 \mathrm{AC}
\end{aligned}
$$

Através das Equações I, J e L observa-se que as frações mássicas das argilas $\mathrm{A}$ e $\mathrm{C}$ contribuem para o aumento de VA, VP e VF. Na Equação J observa-se que os componentes A e C interagem sinergicamente (coeficiente da interação A e C é maior que zero), já na Equação L estes componentes interagem antagonisticamente.

A Tabela IV apresenta os parâmetros estatísticos para as Equações I, J e L ajustadas. Analisando os parâmetros estatísticos principais (Teste F, Valor p, coeficiente de múltipla determinação, $\mathrm{R}^{2}$ e coeficiente de múltipla determinação ajustado, $\mathrm{R}_{\mathrm{A}}^{2}$ ), observa-se que os modelos para VA e VF não são estatisticamente significativos ao nível estipulado (valor $\mathrm{p} \leq$ nível de significância), que o $\mathrm{R}^{2}$ e o $\mathrm{R}_{\mathrm{A}}^{2}$ mostram que os modelos ajustados para VA e VF apresentam variabilidade 
considerável e o modelo ajustado para VP não apresenta variabilidade.

Tabela IV - Estatísticas relevantes para análise de variância das variáveis VA, VP e VF nas equações I, J e L.

[Table IV - Statistics properties relevant for variance analysis of the variables VA, VP and VF in Equations I, $J$ and L.]

\begin{tabular}{cccccc}
\hline Propriedade & Modelo & Teste $\mathrm{F}$ & Valor $\mathrm{p}$ & $\mathrm{R}^{2}$ & $\mathrm{R}_{\mathrm{A}}{ }^{2}$ \\
\hline VA & Quadrático & 4,8653 & 0,0671 & 0,6606 & 0,5248 \\
$\mathrm{VP}$ & Quadrático & 61,7857 & 0,0003 & 0,9611 & 0,9455 \\
$\mathrm{VF}$ & Quadrático & 3,6730 & 0,1044 & 0,5950 & 0,4330 \\
\hline
\end{tabular}

$R^{2}$ : coeficiente de múltipla determinação e $R_{A}^{2}: R^{2}$ ajustado.

Em resumo, a Equação L pode ser tomada como sendo estatisticamente significativa para descrever o comportamento de VP em função das frações mássicas das argilas A e C. As Equações I e J não são estatisticamente significativas.

As Equações $\mathrm{M}, \mathrm{N}$ e $\mathrm{O}$ correlacionando os valores de $\mathrm{VA}, \mathrm{VP}$ e VF com as frações mássicas dos componentes B e C são apresentadas a seguir:

$$
\begin{aligned}
& \mathrm{VA}(\mathrm{cP})=40,37 \mathrm{~B} \\
& \mathrm{VP}(\mathrm{cP})=1,39 \mathrm{C}-2,18 \mathrm{BC} \\
& \mathrm{VF}(\mathrm{mL})=15,71 \mathrm{~B}+21,48 \mathrm{C}
\end{aligned}
$$

Através das Equações $\mathrm{M}, \mathrm{Ne} \mathrm{O}$ observa-se que as frações mássicas das argilas $\mathrm{B}$ e $\mathrm{C}$ contribuem para o aumento de VP e VF, e que o fato do componente $C$ não aparecer na Equação $M$ mostra que ele não contribui para aumentar VA, embora
Tabela V - Estatísticas relevantes para análise de variância das variáveis VA, VP e VF nas Equações $\mathrm{M}, \mathrm{N}$ e $\mathrm{O}$.

[Table $V$-Statistics properties relevant for variance analysis of the variables VA, VP and VF in Equations $M, N$ and $O$.

\begin{tabular}{cccccc}
\hline Propriedade & Modelo & Teste $\mathrm{F}$ & Valor $\mathrm{p}$ & $\mathrm{R}^{2}$ & $\mathrm{R}^{2}$ \\
\hline VA & Quadrático & 0,8745 & 0,4724 & 0,2591 & 0,0000 \\
VP & Quadrático & 21,5797 & 0,0035 & 0,8962 & 0,8546 \\
VF & Linear & 20,3937 & 0,0040 & 0,7727 & 0,7347 \\
\hline
\end{tabular}

$R^{2}$ : coeficiente de múltipla determinação e $R_{A}^{2}: R^{2}$ ajustado.

possa ser importante para determinar outras propriedades da dispersão. Na Equação N observa-se que os componentes B e $\mathrm{C}$ interagem antagonisticamente.

A Tabela $\mathrm{V}$ apresenta os parâmetros estatísticos para as Equações $\mathrm{M}, \mathrm{N}$ e $\mathrm{O}$ ajustadas. Analisando os parâmetros estatísticos principais (Teste F, Valor $\mathrm{p}$, coeficiente de múltipla determinação, $\mathrm{R}^{2}$ e coeficiente de múltipla determinação ajustado, $\mathrm{R}_{\mathrm{A}}^{2}$ ), observa-se que os modelos obtidos para VP e VF são estatisticamente significantes ao nível estipulado (valor $\mathrm{p} \leq$ nível de significância) ao contrário do modelo obtido para $\mathrm{VA}$, que o $\mathrm{R}^{2}$ e o $\mathrm{R}_{\mathrm{A}}^{2}$ mostram que o modelo ajustado para VA apresenta variabilidade considerável, para VP não apresenta variabilidade e para VF uma pequena variabilidade.

Em resumo, as Equações $\mathrm{N}$ e $\mathrm{O}$ podem ser tomadas como sendo estatisticamente significativas para descrever o comportamento de VP e VF em função das frações mássicas das argilas $\mathrm{B}$ e $\mathrm{C}$.

\section{Validação dos modelos}

Com o objetivo de validar os modelos e os resultados obtidos com a resolução das Equações, foram selecionadas

Tabela VI - Composições utilizadas nos testes dos modelos e os respectivos valores observados e previstos de VA, VP e VF.

[Table VI - Compositions used in the models tests and observed and predict values of VA, VP and VF.]

\begin{tabular}{cccccccccc}
\hline & & Argilas & \multicolumn{4}{c}{ Valores observados } & \multicolumn{3}{c}{ Valores previstos } \\
\hline Composições & A & B & C & VA (cP) & VP (cP) & VF (mL) & VA (cP) & VP (cP) & VF $(\mathrm{mL})$ \\
\hline 13 & $1 / 5$ & $4 / 5$ & 0 & 23,7 & 2,0 & 12,8 & 24,1 & 3,3 & 14,5 \\
14 & $4 / 5$ & $1 / 5$ & 0 & 9,5 & 5,0 & 17,5 & 9,5 & 4,7 & 17,5 \\
15 & $1 / 3$ & $2 / 3$ & 0 & 21,4 & 2,8 & 16,2 & 18,8 & 4,4 & 14,3 \\
16 & $2 / 3$ & $1 / 3$ & 0 & 11,2 & 4,5 & 16,2 & 10,7 & 5,2 & 16,0 \\
17 & 0 & $1 / 5$ & $4 / 5$ & 24,0 & 0,5 & 16,4 & 28,6 & 0,8 & 20,4 \\
18 & 0 & $4 / 5$ & $1 / 5$ & 63,2 & 0,0 & 19,2 & 41,9 & 0,0 & 16,9 \\
19 & 0 & $1 / 3$ & $2 / 3$ & 23,0 & 0,5 & 18,8 & 33,8 & 0,5 & 19,6 \\
20 & 0 & $2 / 3$ & $1 / 3$ & 32,3 & 0,0 & 18,2 & 41,2 & 0,0 & 17,6 \\
21 & $1 / 5$ & 0 & $4 / 5$ & 13,0 & 3,5 & 16,8 & 12,1 & 3,0 & 18,5 \\
22 & $4 / 5$ & 0 & $1 / 5$ & 8,2 & 3,5 & 17,5 & 8,0 & 4,0 & 19,0 \\
23 & $1 / 3$ & 0 & $2 / 3$ & 9,3 & 3,8 & 18,8 & 9,7 & 3,6 & 17,9 \\
24 & $2 / 3$ & 0 & $1 / 3$ & 8,3 & 3,8 & 18,5 & 7,5 & 4,2 & 18,1 \\
\hline
\end{tabular}


outras composições. A Tabela VI mostra as formulações de teste e os valores medidos das propriedades que são comparados com os valores preditos pelos modelos representados pelas Equações F, G, H, I, J, L, M, N e O.

Com exceção das formulações binárias contendo as argilas $\mathrm{A}$ e $\mathrm{C}$, as medidas experimentais de $\mathrm{VA}$ encontram-se próximas das estimativas calculadas pelos modelos correspondentes. Para VP e VF observou-se uma pequena variabilidade entre os valores previstos e os valores observados experimentalmente, confirmando o comportamento observado através da análise de variância dos modelos ajustados a estas variáveis.

Em geral, os resultados obtidos com os modelos escolhidos para as formulações binárias, contendo as argilas A e B e as argilas B e C, quando comparados com os resultados obtidos experimentalmente mostram que os modelos são significativamente eficazes para previsão dos resultados das propriedades estudadas.

\section{Influência das composições no comportamento reológico das dispersões}

Comparando os resultados das propriedades reológicas obtidos para as argilas individuais com as normas vigentes para fluidos de perfuração à base de água e argila [17], observa-se que as propriedades não satisfazem às especificações, e dependendo do tipo de argila, obtêm-se sistemas com diferentes graus de floculação.

Os resultados reológicos das dispersões preparadas coma argila A evidenciaram um estado parcialmente defloculado. Segundo Amorim [18], este estado pode ser definido como um estado intemediário entre os estados defloculado e floculado, no qual as partículas estão dispersas no meio líquido com poucas interações elétricas e de massa. Em outras palavras, as forças atrativas entre as partículas de argila, que promovem a sua aglomeração, foram parcialmente vencidas pelas forças repulsivas originárias da transformação da argila policatiônica em sódica.

Os resultados reológicos das dispersões preparadas com as argilas B e C, respectivamente, apresentam-se no estado floculado-gel, caracterizado pelos elevados valores de VA e baixos valores de VP. Neste caso, a concentração de $\mathrm{Na}_{2} \mathrm{CO}_{3}$ é suficientemente alta e promove o aumento na intensidade das interações elétricas entre as partículas, bem como novas interações entre as partículas [18].

Para os sistemas formados por misturas com as argilas A, $\mathrm{B}$ e $\mathrm{C}$, as mudanças nos parâmetros reológicos e no estado de floculação/defloculação do sistema podem ser explicadas através das interações entre as partículas do sistema formado com as argilas individuais.

A Fig. 1 mostra a variação das propriedades estudadas em função das formulações binárias das argilas A e B, apresentando os dados obtidos experimentalmente.

Visualiza-se, em termos gráficos, a influência de cada argila sobre as propriedades reológicas VA e VP e de filtração VF. Observa-se que o aumento da quantidade da argila A na formulação proporciona o aumento de $\mathrm{VP}$ e de VF e a diminuição de VA, efeito inverso é observado para o aumento da quantidade da argila B na mistura. O que já foi confirmado através da análise estatística.

Com o aumento da argila A, no sistema formado pela mistura A e B, ocorre a passagem do estado floculado-gel para o estado parcialmente defloculado, caracterizado pela queda de VA.

As formulações $(\mathrm{A}, \mathrm{B})=(1 / 4,3 / 4)$ e $(\mathrm{A}, \mathrm{B})=(1 / 2,1 / 2)$ atendem às restrições para os parâmetros reológicos VAe VP e de filtração VF apresentadas pela norma da Petrobras [17] para fluidos à base de água e argila utilizados na perfuração de poços de petróleo.

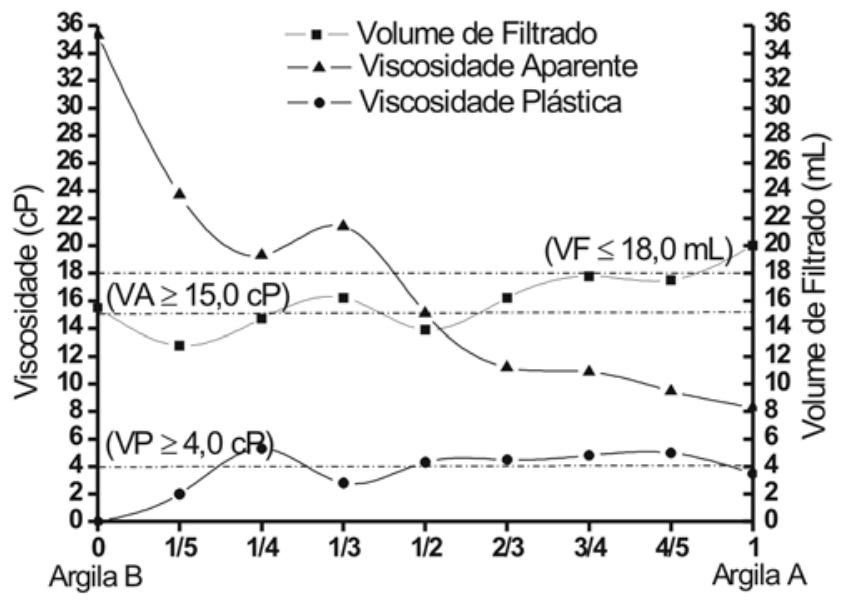

Frações Mássicas

Figura 1: Variação das propriedades reológicas VA e VP e de filtração VF em função da composição (argila A + argila B).

[Figure 1: Variation of rheological properties VA and VP and of filtration VF in function of the composition (clay $A+$ clay $B)$.

A Fig. 2 mostra a variação das propriedades estudadas em função das formulações binárias das argilas A e C, apresentando os dados obtidos experimentalmente.

Visualiza-se, em termos gráficos, a influência de cada argila sobre as propriedades reológicas VA e VP e de filtração VF. Observa-se que um aumento da quantidade da argila A na mistura proporciona o aumento de VP e de VF e a diminuição de VA, efeito inverso é observado para o aumento da quantidade da argila $\mathrm{C}$ na mistura. $\mathrm{O}$ que já foi confirmado através da análise estatística.

Com o aumento da argila A no sistema ocorre a mudança do estado floculado-gel para o estado parcialmente defloculado, com a diminuição das interações elétricas entre as partículas provocadas pelo aumento da quantidade de A, para um estado de menor floculação, caracterizado pela diminuição nos valores de VA.

As formulações binárias das argilas A e C não atendem às restrições para os parâmetros reológicos VA e VP e de filtração VF apresentadas pela norma da Petrobras [17] para fluidos à base de água e argila utilizados na perfuração de poços de petróleo. 


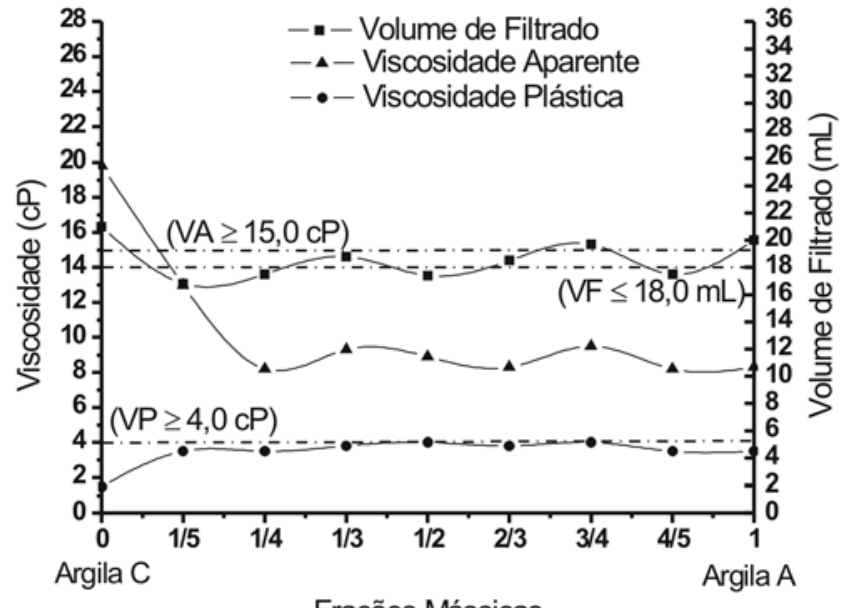

Frações Mássicas

Figura 2: Variação das propriedades reológicas VA e VP e de filtração VF em função da composição (argila A + argila C).

[Figure 2: Variation of rheological properties VA and VP and of filtration $V F$ in function of the composition (clay $A+$ clay $C$ ).]

A Fig. 3 mostra a variação das propriedades estudadas em função das formulações binárias das argilas $\mathrm{B}$ e $\mathrm{C}$, apresentando os dados obtidos experimentalmente.

Visualiza-se, em termos gráficos, a influência de cada argila sobre as propriedades reológicas VA e VP e de filtração VF. Observa-se que um aumento da quantidade da argila B na mistura proporciona o aumento de VA. VP não apresenta variabilidade considerável.

Este sistema é formado por duas argilas (B e C) que, individualmente, apresentam dispersões no estado floculadogel. Para determinadas proporções a mistura das argilas B e C proporcionam um grau bastante elevado de floculação do sistema dificultando a previsão quantitativa das propriedades

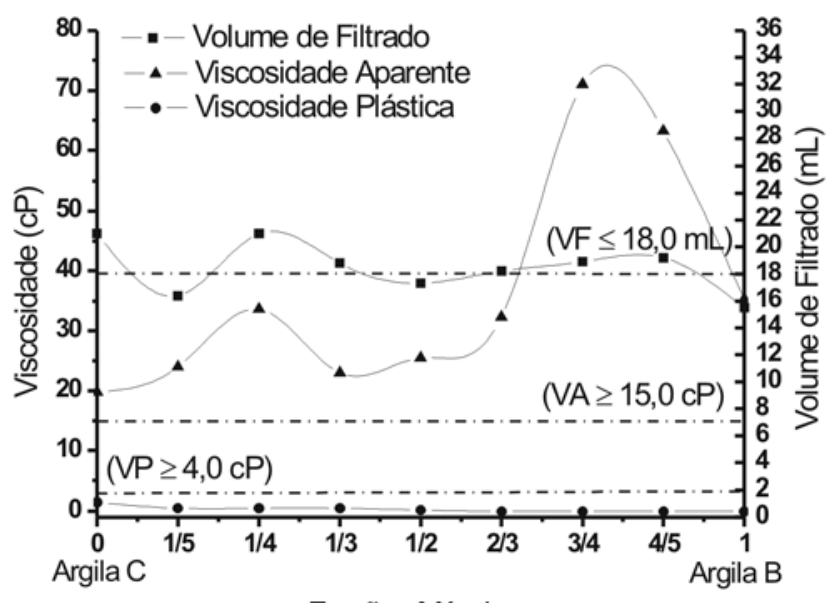

Frações Mássicas

Figura 3: Variação das propriedades reológicas VA e VP e de filtração VF em função da composição (argila $\mathrm{B}+\operatorname{argila~C}$ ).

[Figure 3: Variation of rheological properties VA and VP and of filtration VF in function of the composition (clay $B+$ clay $C)$. reológicas através do delineamento de misturas. Quando a proporção de argila B na mistura aumenta o sistema tende a torna-se mais floculado.

As formulações binárias das argilas B e C não atendem às restrições para os parâmetros reológicos VA e VP e de filtração VF apresentadas pela norma da Petrobras [17] para fluidos à base de água e argila utilizados na perfuração de poços de petróleo.

Otimização da combinação das argilas estudadas para obtenção de bentonitas sódicas que favorecem a melhoria das propriedades reológicas

Através das Figs. 1, 2 e 3, observa-se que apenas as formulações compostas pelas misturas binárias das argilas A e B, nas proporções estudadas, apresentaram resultados para as propriedades reológicas que atendem às restrições impostas para fluidos de perfuração de poços de petróleo. Portanto, esta mistura binária foi escolhida para os estudos de otimização matemática.

A solução do sistema de inequações formado pelas Equações de regressão F, G e H e pelas restrições (Equações C, D e E), conduziu a composições que, de acordo com os modelos matemáticos escolhidos, são adequadas para produzir dispersões com as características pretendidas.

A solução para o sistema formado resultou nas seguintes faixas de composições que atendem às restrições para as propriedades reológicas VA e VP e de filtração VF: fração mássica da argila A maior ou igual a 0,33 e menor ou igual a $0,45(0,33 \leq \mathrm{A} \leq 0,45)$ e, consequentemente, fração mássica da argila $\mathrm{B}$ maior ou igual a 0,55 e menor ou igual a 0,63 $(0,55 \leq \mathrm{B} \leq 0,67)$.

\section{CONCLUSÕES}

A utilização do delineamento de misturas, com o objetivo de modelar matematicamente as variáveis VA, VP e VF de dispersões aquosas de bentonitas, mostrou-se adequada para dispersões sendo possível a obtenção de modelos matemáticos, estatisticamente significativos, que relacionam VA, VP e VF com as proporções das argilas na mistura. Apresentando uma limitação para casos em que as dispersões apresentaram-se no estado floculado-gel.

Os parâmetros reológicos estão diretamente relacionados com a composição das misturas analisadas e as interações entre as partículas dos sistemas formados com as misturas das argilas estudadas dependem fortemente do comportamento reológico dos sistemas formados com as argilas individuais. A mistura de argilas que apresentam dispersões no estado floculado-gel proporciona um efeito aditivo na viscosidade, impedindo que os dados experimentais se aproximem dos dados previstos pelos modelos matemáticos, que por sua vez, não são estatisticamente significativos.

A solução simultânea das equações de regressão, sujeitas a restrições impostas à VA, VP e VF permitiu delimitar uma faixa de composições (Argilas A e B: $0,33 \leq \mathrm{A} \leq 0,45$ e $0,55 \leq \mathrm{B} \leq 0,67$ ) que favorece a melhoria das propriedades 
reológicas de argilas bentoníticas sódicas para fluidos à base de água e argila utilizados na perfuração de poços de petróleo.

\section{AGRADECIMENTOS}

À CAPES e ao CNPq (Processo Nº DCR 309873/2003-7) pelo apoio financeiro através da concessão de bolsas.

Ao LABDES - Laboratório de Referência em Dessalinização, pelo uso de seus laboratórios e apoio à pesquisa.

\section{REFERÊNCIAS}

[1] J. A. Cornell, "Experiments with mixtures-designs, models, and the analysis of mixtures data", $2^{\text {nd }}$ Ed., Wiley, New York (1990) 19.

[2] B. Barros Neto, I. E. Scarminio, R. E. Bruns, "Planejamento e otimização de experimentos", $2^{a}$ Ed., Editora Unicamp, S. Paulo (1996) 187.

[3] C. Reis, J. C. Andrade, Química Nova 19, 3 (1996) 313.

[4] I. G. Branco, C. A. Gasparetto, Ciênc. Tecnol. Aliment., Campinas 23 (2003) 166.

[5] S. L. Correia, D. Hotza, A. M. Segadães, Anais do Congresso em Ciência dos Materiais do Mercosul, Sulmat, Joinville, SC (2004).

[6] L. M. Schabbach, A. P. N. Oliveira, M. C. Fredel, D. Hotza, Am. Ceram. Soc. Bull. 82 (2003) 47.

[7] C. M. Gomes, "Avaliação do comportamento reológico de suspensões cerâmicas triaxiais utilizando abordagem do delineamento de misturas", Dissertação de Mestrado, UFSC, Florianópolis (2004).
[8] A. M. Bernardim, F. R. Pereira, H. G. Riella, Anais do $44^{\circ}$ Congresso Brasileiro de Cerâmica, S. Paulo, SP (2000) 19601.

[9] L. C. Chiari, A. O. Boschi, Anais do $44^{\circ}$ Congresso Brasileiro de Cerâmica, S. Paulo, SP (2000) 20801.

[10] C. R. Oliveira, J. Castral Jr., C. V. Santos, C. Bolfarini, L. C. Chiari, Anais do $44^{\circ}$ Congresso Brasileiro de Cerâmica, S. Paulo, SP (2000) 19001.

[11] J. Alexandre, R. Toledo, F. Saboya, M. Pereira, Anais do $44^{\circ}$ Congresso Brasileiro de Cerâmica, S. Paulo, SP, (2000) 50301.

[12] J. Alexandre, R. Toledo, F. Saboya, M. Pereira, Cerâmica 47, 303 (2001) 170.

[13] P. Souza Santos, Ciência e Tecnologia de Argilas, $2^{a}$ Ed, vol. 3. Ed. Edgar Blücher, S. Paulo, SP (1989) 35.

[14] F. R. V. Díaz, P. Souza Santos, H. S. Santos, Química Industrial 42 (1992) 33.

[15] L. F. A. Campos, "Reologia de misturas de esmectitas tratadas com aditivos primários e secundários para obtenção de fluidos de perfuração", Plano de Tese, Doutorado em Engenharia de Processos, CCT/UFCG, Campina Grande, PB (2003).

[16] D. V. Viana, "Dimensionamento de misturas de argilas bentoníticas de Boa Vista, PB visando seu uso em fluidos de perfuração de poços de petróloeo", Relatório Final PIBIC/ CNPq (2004).

[17] Petrobrás, "Ensaio de viscosificante para fluido de perfuração à base de água na exploração e produção de petróleo", Método, N-2605 (1998).

[18] L. V. Amorim, "Melhoria, proteção e recuperação da reologia de fluidos hidroargilosos para uso na perfuração de poços de petróleo", Tese de Doutorado em Engenharia de Processos, CCT/UFPB, Campina Grande, PB (2003).

(Rec. 15/08/2005, Ac. 11/11/2005) 